\title{
A simple method for semi-random DNA amplicon fragmentation using the methylation-dependent restriction enzyme MspJ
}

Hiroshi Shinozuka ${ }^{1,2}$, Noel O I Cogan ${ }^{1,2}$, Maiko Shinozuka ${ }^{1,2}$, Alexis Marshall ${ }^{1,2}$, Pippa Kay ${ }^{1}$, Yi-Han Lin ${ }^{1,2}$, German C Spangenberg ${ }^{1,2,3}$ and John W Forster ${ }^{1,2,3^{*}}$

\begin{abstract}
Background: Fragmentation at random nucleotide locations is an essential process for preparation of DNA libraries to be used on massively parallel short-read DNA sequencing platforms. Although instruments for physical shearing, such as the Covaris S2 focused-ultrasonicator system, and products for enzymatic shearing, such as the Nextera technology and NEBNext dsDNA Fragmentase kit, are commercially available, a simple and inexpensive method is desirable for high-throughput sequencing library preparation. MspJl is a recently characterised restriction enzyme which recognises the sequence motif CNNR (where $R=G$ or $A$ ) when the first base is modified to 5-methylcytosine or 5-hydroxymethylcytosine.
\end{abstract}

Results: A semi-random enzymatic DNA amplicon fragmentation method was developed based on the unique cleavage properties of MspJl. In this method, random incorporation of 5-methyl-2'-deoxycytidine-5'-triphosphate is achieved through DNA amplification with DNA polymerase, followed by DNA digestion with MspJ. Due to the recognition sequence of the enzyme, DNA amplicons are fragmented in a relatively sequence-independent manner. The size range of the resulting fragments was capable of control through optimisation of 5-methyl-2'-deoxycytidine-5'triphosphate concentration in the reaction mixture. A library suitable for sequencing using the lllumina MiSeq platform was prepared and processed using the proposed method. Alignment of generated short reads to a reference sequence demonstrated a relatively high level of random fragmentation.

Conclusions: The proposed method may be performed with standard laboratory equipment. Although the uniformity of coverage was slightly inferior to the Covaris physical shearing procedure, due to efficiencies of cost and labour, the method may be more suitable than existing approaches for implementation in large-scale sequencing activities, such as bacterial artificial chromosome (BAC)-based genome sequence assembly, pan-genomic studies and locus-targeted genotyping-by-sequencing.

Keywords: Second-generation sequencing technology, Genotyping-by-sequencing, 5-methylcytosine, Restriction endonuclease, DNA shearing

\footnotetext{
* Correspondence: john.forster@ecodev.vic.gov.au

'Department of Economic Development, Jobs, Transport and Resources,

Biosciences Research Division, AgriBio, Centre for AgriBioscience, 5 Ring

Road, La Trobe University Research and Development Park, Bundoora,

Victoria 3083, Australia

${ }^{2}$ Dairy Futures Cooperative Research Centre, Bundoora, Australia

Full list of author information is available at the end of the article
} 


\section{Background}

Massively parallel short-read sequencing technologies have become commonly used not only for de novo genome sequencing, but also for a wide range of biological purposes, such as resequencing and large-scale genotyping studies. Fragmentation at random nucleotide locations is an essential component of library construction for the various short-read sequencing instruments [1], through delivery of multiple read initiation points in template molecules. Sequence information may then be decoded through computational assembly of the short reads. Physical shearing is recommended by the manufacturers of all secondgeneration massively parallel DNA sequencing systems, due to the high reproducibility and randomness of fragmentation. However, the process is likely to require the use of dedicated instruments. The Nextera technology (Illumina, California, USA) and the NEBNext dsDNA Fragmentase kit (New England Biolabs, Massachusetts, USA) are alternative random DNA fragmentation methods which require only standard laboratory instruments [2-4]. The Nextera technology uses a transposon-transposase combination for random fragmentation of template DNA and attachment of transposon ends at the cleaved sites, permitting subsequent PCR amplification and sequencing. With the NEBNext dsDNA Fragmentase kit, double-stranded template DNA is fragmented in two sequential steps: nicks are enzymatically introduced into DNA, which is then cleaved at the nicked sites. These enzyme-based methods, however, require DNA sample preparation (buffer replacement and DNA concentration adjustment) for effective digestion, and the size of products is sensitive to both DNA sample quality and reaction duration, all of which require optimisation for each sample in order to achieve the desired outcome.

MspJI is a recently characterised modification-dependent endonuclease [5]. The enzyme was isolated from Mycobacterium sp. JLS and recognises sites containing the sequence motif CNNR ( $\mathrm{R}=\mathrm{G}$ or A nucleotides) when the first base is a 5-methylcytosine $\left(5^{\mathrm{m}} \mathrm{C}\right)$ or 5-hydroxymethylcytosine, and cleaves DNA at $\mathrm{N}_{12} / \mathrm{N}_{16}$ bases distant from the modified cytosine on the 3'-side. Enzyme activity is enhanced by short double-stranded DNA that includes the MspJI recognition site (and so acts as an enzyme activator). Digestion of genomic DNA with the MspJI enzyme generates fragments 3234 bp in length, containing ${ }^{\mathrm{m}} \mathrm{CpG}$ or ${ }^{\mathrm{m}} \mathrm{CNG}$ sites central to the fragment. Methylation status of the human genome has been analysed through sequencing of such fragments [6]. Due to these unique features as a methylation-dependent restriction enzyme with adjacent non-specific cleavage activity, MspJI is expected to be highly useful for DNA modification and epigenomic studies [7].

FspEI and LpnPI are also recently characterised modification-dependent endonucleases, derived from
Frankia sp. EAN1pec and Legionella pneumophila Philadelphia 1, respectively [5]. The sequence recognition sites for FspEI and LpnPI are 5'-CC-3' and 5'CCDG-3' ( $\mathrm{D}=\mathrm{A}, \mathrm{G}$ or $\mathrm{T}$ nucleotides), respectively, when the second base is a $5{ }^{\mathrm{m}} \mathrm{C}$ or 5 -hydroxymethylcytosine. Similar to MspJI, activity of the two enzymes is stimulated by short DNA templates containing the recognition site, and the enzymes produce DNA fragments with 5'- termini including cohesive ends. These two enzymes hence provide potential alternatives to MspJI as a tool for DNA fragmentation.

The present study describes a simple and inexpensive method for generation of semi-randomly fragmented DNA from amplicon templates. DNA amplicons with randomlyincorporated 5-methyl-2'-deoxycytidine-5'-triphosphate (5methyl-dCTP) were synthesised with DNA polymerase, and then digested with the MspJI restriction enzyme. The size range of the MspJI-digested fragments was capable of control through optimisation of 5-methyl-dCTP concentration. A purification procedure is unnecessary for DNA digestion with MspJI, which permits high-throughput sequencing library preparation. Short DNA fragments were also generated from a range of templates with a whole genome amplification kit based on activity of the Ф29 DNA polymerase, using the same methodology. Illumina sequencing libraries with inserts of 200 or $550 \mathrm{bp}$ in length were successfully prepared using the MspJI-digested DNA, and were processed on the Illumina MiSeq platform.

\section{Results and discussion}

\section{DNA amplification with 5-methyl-dCTP and MspJ digestion}

PCR amplification in the presence of 5-methyl-dCTP was performed with locus-specific primers and Agrobacterium tumefaciens genomic DNA (Agro gDNA) as template (Additional file 1). No significant difference in yield of PCR amplicon due to 5-methyl-dCTP concentration levels (final concentrations of 2, 4, or $8 \mu \mathrm{M}$ in the PCR solution) was observed, based on the results of agarose gel electrophoresis, which was consistent with the previous studies (Figure 1a) [8,9]. The PCR amplicons of the Agro_gc40, Agro_gc50 and Agro_gc60 sequences, of which G/C content ratios were 40, 50 and $60 \%$, respectively, were digested using the MspJI enzyme to identify the consequence of $5{ }^{\mathrm{m}} \mathrm{C}$-containing DNA cleavage in the size-resolution range afforded by a $2.5 \%(\mathrm{w} / \mathrm{v})$ agarose gel (Figure 1b). The size range of the digested DNA largely depended on concentration of 5-methyl-dCTP in the amplification solution, such that a lower range was obtained by a higher 5-methyl-dCTP concentration. Size range was also related to $\mathrm{G} / \mathrm{C}$ content ratio, such that smaller DNA fragments were identified when amplicons with a higher ratio were digested (Figure 1b). This is probably due to the presence of at least one $\mathrm{C}-\mathrm{G}$ base pair in 


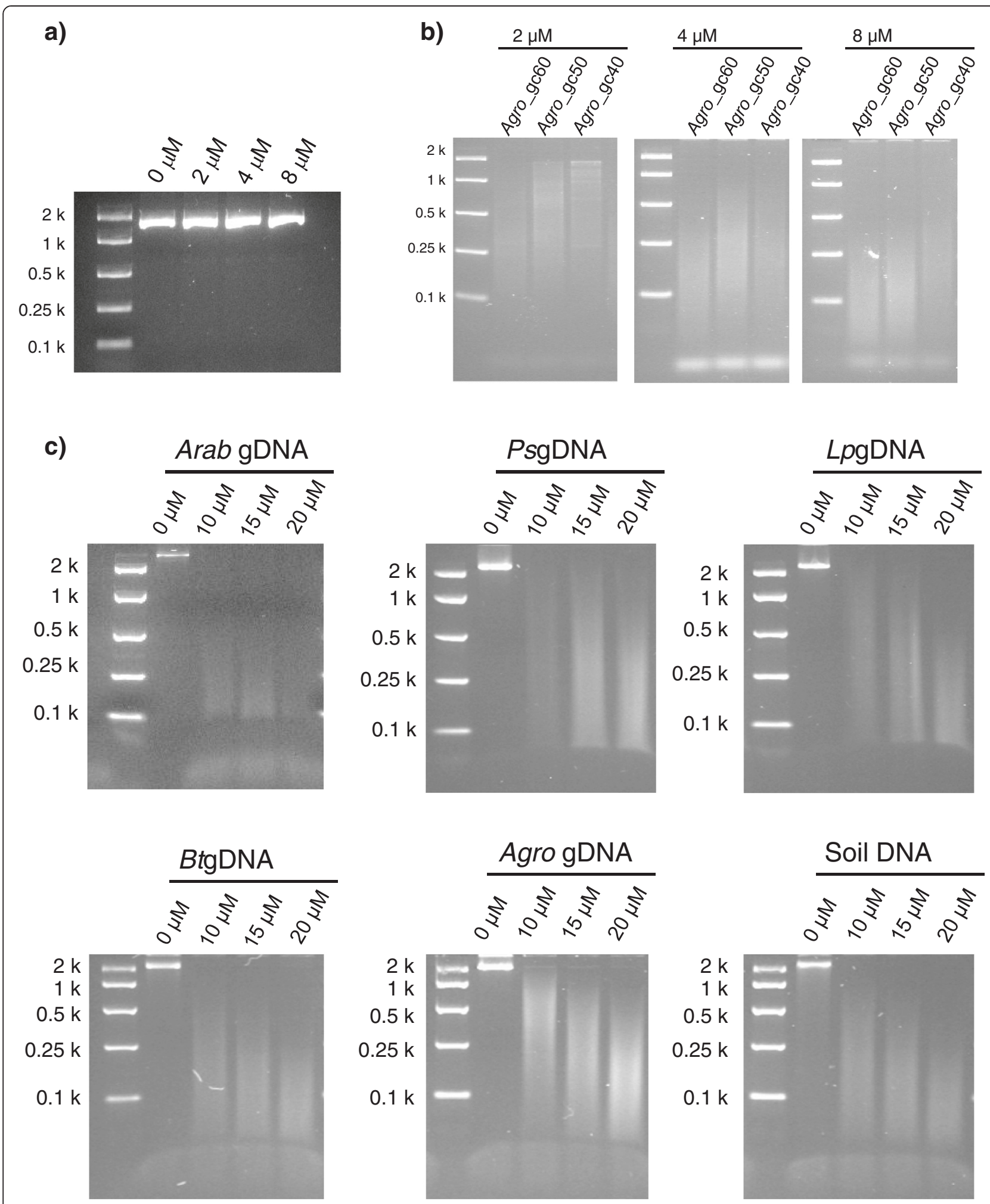

Figure 1 Mspl-enzymatic digestion of $5^{\mathrm{m} C}$-containing PCR and $\Phi 29$ products. (a) DNA fragments amplified with the locus-specific PCR primers for the Agro_gc50 sequence under the presence of 5-methyl-dCTP (0, 2, 4 or $8 \mu \mathrm{M})$. (b) MspJ-digested DNA fragments derived from PCR products with each locus-specific primers and Agro gDNA as DNA template. Molar concentration denotes the 5-methyl-dCTP-concentration in PCR solution. (c) MspJ-enzymatic digestion of $\Phi 29$ enzyme-amplified DNA with randomly incorporated $5^{\mathrm{m} C}$ from a range of DNA templates. 0, 10, 15 and $20 \mu \mathrm{M}$ denote final concentrations of 5-methyl-dCTP in the REPLI-g WGA mixture. 
the MspII recognition site $\left(5^{\mathrm{m}} \mathrm{CNNR}\right)$, which will produce a small bias in incidence toward regions of higher G/C content ratio.

The Agro_gc50 sequence was also amplified with four types of DNA polymerase. The sequence was successfully amplified with all polymerases in the presence of 5-methyl$\mathrm{dCTP}$, and the amplicons were digested with MspJI. No significant differences in size range were observed, suggesting that a variety of DNA polymerases may be used for the proposed DNA fragmentation method (Additional file 2). A further characterisation of MspJI enzymatic activity indicated that components of the PCR solution do not significantly affect activity of the MspJI enzyme when diluted in the reaction mixture; the MspJI-mediated digestion of amplicons is completed within 4 hours; and the digestion result is independent of input DNA amount, when performed in an appropriate volume of reaction mixture (Additional file 3).

Whole genome amplification (WGA) was performed using the QIAGEN REPLI-g mini kit in the presence of 5-methyl-dCTP (10 to $20 \mu \mathrm{M}$ ) with genomic DNA samples from Arabidopsis thaliana (L.) Heynh. ecotype Columbia (Arab gDNA), a field pea (Pisum sativum L. subsp. sativum var. arvense (L.) Poir.) genotype (PsgDNA), a perennial ryegrass (Lolium perenne L.) genotype (LpgDNA), a bovine (Bos taurus L.) genotype (BtgDNA), Agro gDNA, and a DNA sample from soil harvested in South Australia (Soil DNA) as templates. Amplified product was visualised on an agarose gel, revealing no significant differences in DNA amplification due to variation of 5-methyl-dCTP concentration. The amplified DNA was digested with MspJI (Figure 1c). Similar size distribution patterns were detected across varying 5-methyl-dCTP concentrations: a majority of DNA fragments from the $20 \mu \mathrm{M}$ 5-methyl-dCTP-containing solutions was shorter than $250 \mathrm{bp}$, and a proportion of DNA fragments from $10 \mu \mathrm{M}$ 5-methyl-dCTP-containing solutions was close to $1,000 \mathrm{bp}$, or larger, in size.

\section{Massively parallel sequencing of MspJl-digested templates}

For exemplification of the effects on distribution of fragments generated with the present method, sequencing libraries were prepared from Agro gDNA and Arab gDNA, using the MspJI-based and standard physical fragmentation methods (Additional file 4). The libraries were sequenced on the Illumina MiSeq platform. Totals of 1,380,029 and 1,219,389 reads derived from $\Phi 29$ enzyme amplification were aligned with the Agrobacterium circular and linear chromosomes (Figure 2a, Table 1). From the physically sheared DNA, totals of 546,292 and 496,662 reads were aligned with the circular and linear chromosomes, respectively. When the short reads from the MspJI-digested and physically sheared DNA were aligned, $99.99 \%$ and $99.8 \%$ of the reference Agrobacterium genome sequence was covered, respectively. When the sequencing reads derived from Arab gDNA were aligned with the reference sequence, 3-5 million reads were assigned to each chromosome (Figure $2 b$, Table 1). Average depths of coverage from the enzymatically fragmented and physically sheared libraries were 43.3 and 53.3 times, respectively, which covered $97.9 \%$ and 99.3\% of the reference Arabidopsis genome sequence. The results of resequencing activities suggested that MspJI successfully generates DNA templates with a moderate level of random fragmentation.

For a further exemplification of the effects on distribution of fragments generated with a methylation-dependent restriction enzyme, the BtKIT1-10 and BtKIT27-37 sequences were amplified from BtgDNA, using the Roche Expand Long Range dNTPack kit in the presence of 7.5, 15 and $60 \mu \mathrm{M}$ 5-methyl-dCTP (Additional file 1). Amplicons from the 7.5, 15 and $60 \mu \mathrm{M}$ 5-methyl-dCTP reactions were digested with MspJI, FspEI or LpnPI (Additional file 5). Illumina sequencing libraries were prepared from the enzyme-digested DNA and sequenced on the MiSeq platform (Additional file 4). Totals of 3566-10,972 reads were aligned with the reference sequences, covering each nucleotide position of the reference. CVs for each data set were between 0.29 and 1.11 (Figure 3). The read alignment result indicated that the read distribution from the LpnPI-digested library was more skewed than those from the other libraries, and there was no large difference in the CVs between the MspJI- and FspEIdigested libraries. The average frequencies of potential MspJI-, FspEI- and LpnPI-recognition sites were once in every 4,8 and $51.2 \mathrm{bp}$, respectively. This alignment result suggests that although the read distribution pattern depends on the frequency of the recognition sites, an incidence of once in every $8 \mathrm{bp}$ is sufficient to generate reads with relatively even distribution.

\section{Potential application 1: sequencing of BAC clones}

Although high-throughput DNA sequencing technologies have delivered a cost-efficient whole-genome shotgun sequencing method for those species with large genome sizes, information from BAC-based genomic libraries is valuable for effective DNA sequence assembly [10]. Sequence information from BAC-ends is commonly used for de novo assembly of large genomes [11,12]. The BAC-end sequencing procedure, however, requires a large investment, as it depends on the Sanger sequencing method [13]. A simple sequencing method for BAC clones using high-throughput sequencing technologies is described here.

BAC DNA was amplified in the reaction mixture of the REPLI-g mini kit, in which $30 \mu \mathrm{M} 5$-methyl-dCTP were included (Figure 4a, Additional file 6). The amplicons were digested with MspJI. A sequencing library was 


\section{a)}

Agrobacterium circular chromosome

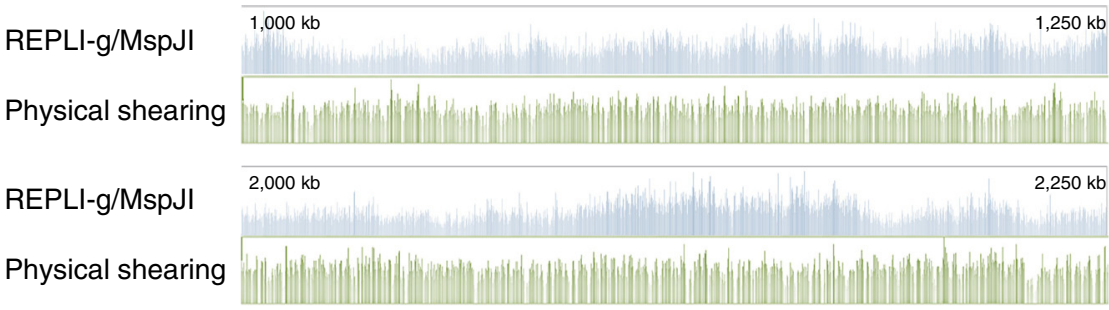

Agrobacterium linear chromosome

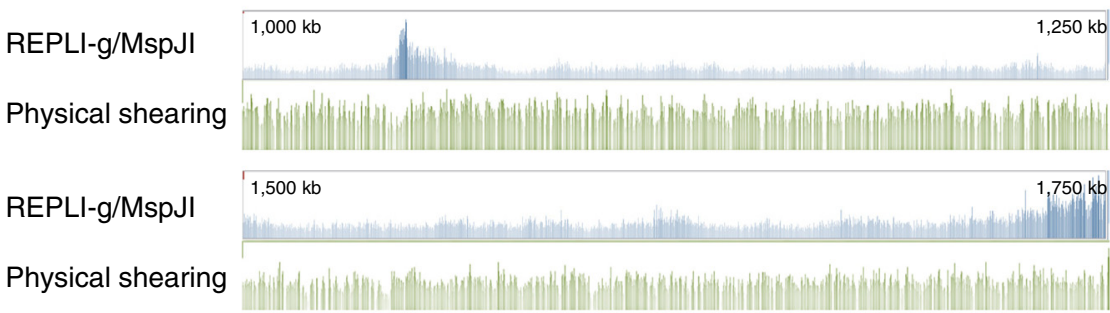

b)


Figure 2 Illumina MiSeq short read-sequencing results of the libraries constructed from MspJ-digested and physically sheared DNA. The sorted alignment was visualised using the Tablet viewers. (a) Alignment results of $\Phi 29$ enzyme-amplified Agro gDNA-derived reads and physically sheared Agro gDNA-derived reads to the reference Agrobacterium genome sequences for the 1,000-1,250 and 2,000-2250 kb regions of the circular and linear chromosomes. (b) Alignment results of the Arabidopsis genome with the MspJ-enzymatic fragmentation and physical shearing methods. Read coverage depth for the 10,000-10,250, 20,000-20,250 and 30,000-30,250 kb regions of chromosome 1 was visualised.

prepared from the MspJI-digested DNA and processed on the Illumina MiSeq platform to generate 395,498558,276 reads for each sample. Totals of $27,585-86,861$ reads (7.0-15.6\%) were aligned with the reference, which covered over $99.6 \%$ of the reference sequences (Figure $4 \mathrm{~b}$, Table 2). The alignment result indicated that $81 \%-86 \%$ of reads were derived from the Escherichia coli (E. coli) genome. When a subset of 100,000 reads was aligned, around
$99 \%$ of the reference BAC insert sequences were covered an average redundancy of 21-27 times. A single run of the Illumina MiSeq platform may generate up to 50 million reads, permitting individual sequencing of a whole 384-well plate of BAC clones with moderate depth of read coverage. With the Illumina MiSeq platform, a single clone may be analysed at the cost of US\$7.2 (Additional file 7). 
Table 1 Result of Agrobacterium and Arabidopsis genome resequencing using the two different DNA fragmentation methods

\begin{tabular}{llllll}
\hline Species & Fragmentation method & Chromosome & Number of reads & Ratio of coverage (\%) & Average coverage depth (time) \\
\hline Agrobacterium & REPLI-g/MspJI & Circular & $1,380,029$ & 99.9 & 64.3 \\
& & Linear & $1,219,389$ & 99.9 & 77.8 \\
& Physical shearing & Circular & 546,292 & 99.8 & 27.4 \\
& & Linear & 496,662 & 99.9 & 34.1 \\
Arabidopsis & 1 & $4,611,463$ & 97.3 & 36.4 \\
& REPLI-g/MspJI & 2 & $4,759,134$ & 98 & 47.7 \\
& & 3 & $4,702,657$ & 98.3 & 39.9 \\
& & 4 & $3,088,231$ & 98 & 37.8 \\
& & 5 & $4,222,266$ & 98 & 41.5 \\
& & & $5,116,095$ & 98.9 & 75 \\
\end{tabular}

Ratio of coverage denotes the value for specific nucleotides located on each chromosome.

Potential application 2: whole genome amplification and sequencing of bacterial and fungal genomes

High-throughput DNA sequencing technologies also provide an efficient method for pan-genome studies, especially for bacterial and fungal species [14,15]. Due to high levels of genomic diversity, a substantial number of bacteria or fungal strains must, however, be sequenced in order to define both core- and pan-genome constituents, and so a high-throughput library preparation method is required. Previously, direct WGA from fungal tissues was suggested as an efficient DNA sample preparation method [16]. A combination of the WGA and MspJI-based DNA fragmentation methods may permit high-throughput library processing.

Genomic DNA from the perennial ryegrass-associated endophyte (Epichloë festucae var. lolii syn. Neotyphodium lolii) was amplified from a section of fungal mycelium (Figure 5a). In the multiple displacement amplification (MDA) reaction mixture, $15 \mu \mathrm{M}$ 5-methyl-dCTP was included, and the amplified DNA was digested with MspJI (Figure 5b). A sequencing library was prepared from the MspJI-digested DNA and processed on the Illumina MiSeq platform. Totals of 2.7 million reads were generated, and 81,237 and 59,445 reads were aligned with the reference contig, which represent 1.6 and $1.3 \mathrm{Mb}$ sections,

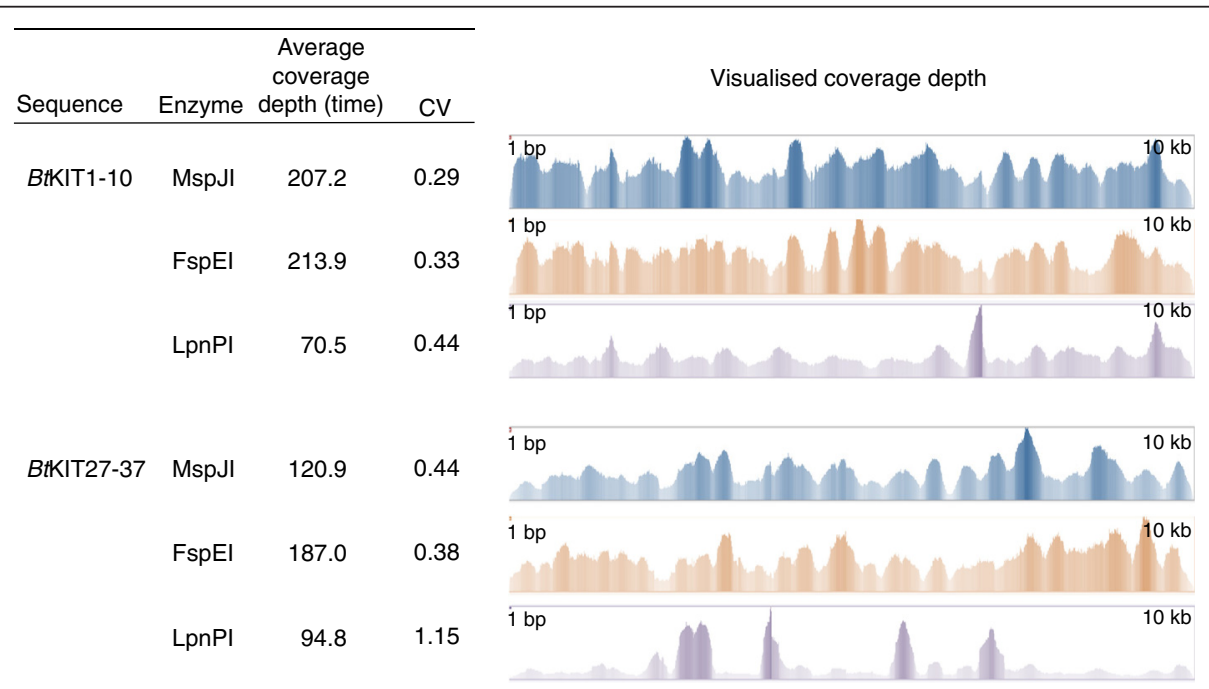

Figure 3 Short read-sequencing results of the libraries constructed from MspJl, FspEl and LpnPI-digested DNA. Read coverage depth was visualised using the Tablet viewer. 


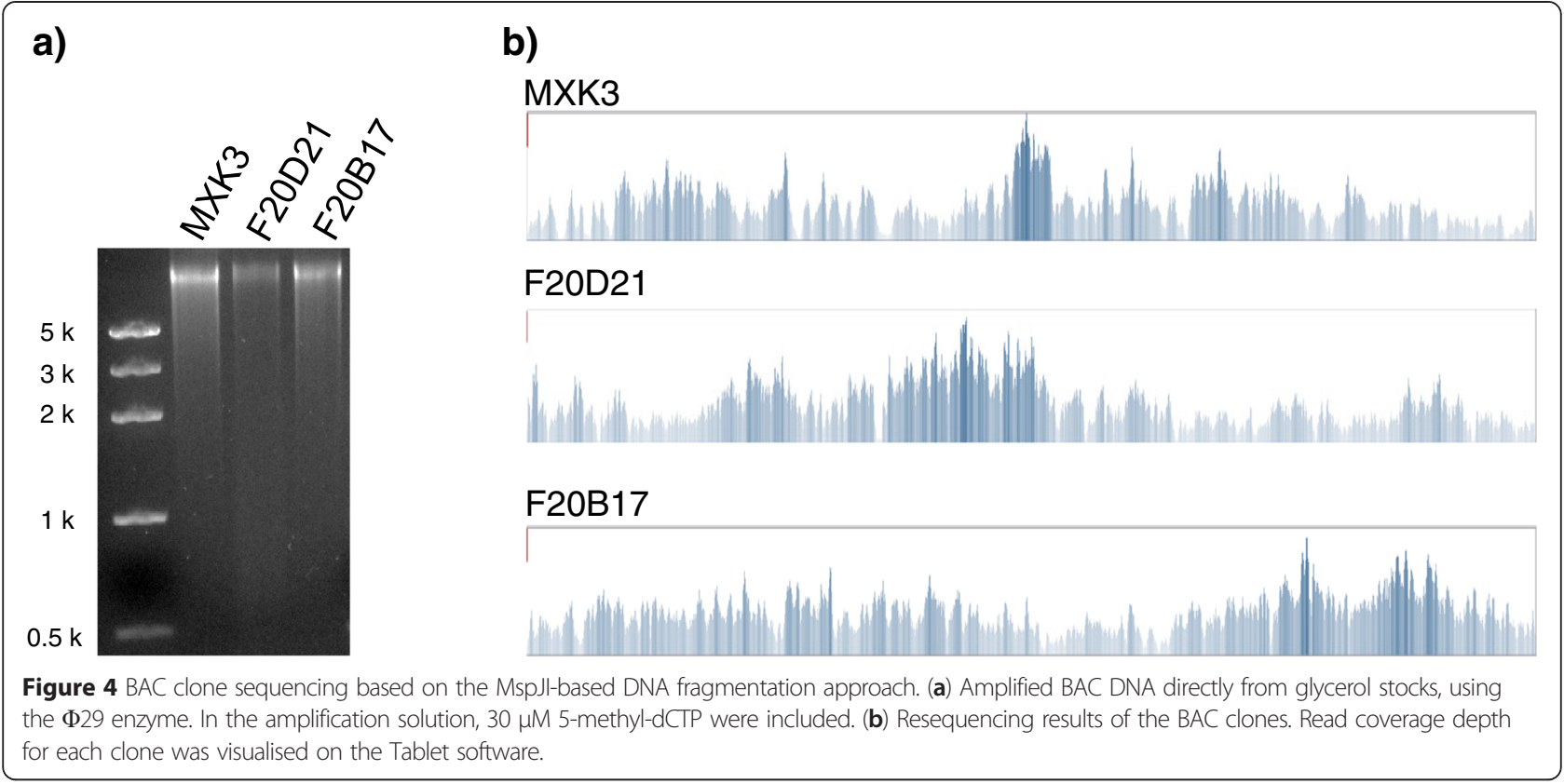

respectively, of a genome $30 \mathrm{Mb}$ in length (Table 3). A previous study reported that WGA was successfully achieved from as few as 24 fungal spores, which contribute to significantly reduced durations of DNA sample preparation [16]. DNA fragmentation with MspJI does not require several procedures subsequent to WGA, and so may enhance efficiency of the WGA-based sequencing library preparation (Figure $5 \mathrm{c}$ ).

\section{Potential application 3: sequencing of PCR amplicons}

Massively parallel sequencing technologies have permitted whole genome re-sequencing in a cost-effective manner [17]. Subsequently, genome-wide association studies (GWASs) have identified DNA polymorphisms that are correlated with trait-specific variation [18]. The numbers of relevant DNA polymorphisms identified through GWASs have, however, been relatively small $[17,18]$. Identification of trait locus variation-related DNA polymorphisms could hence be usefully followed by conversion into specific PCR-based markers, permitting locus-targeted genotyping over larger numbers of individuals $[19,20]$.
Through PCR with 5-methyl-dCTP, candidate sequences for genes involved in the perennial ryegrass flowering signaling pathway were amplified from the DNA samples of the p150/112 $\mathrm{F}_{1}$ genetic mapping population (Figure 6a) [21]. The PCR amplicons were digested with MspJI, and sequencing library was prepared for the Illumina MiSeq platform (Figure 6b, Additional file 8). The sequencing reads were aligned against the references, and SNPs, which could be utilised for genetic linkage mapping studies, were identified in 7 of the sequences (Additional file 9). In the $L p C O$ and $L p F T$ sequence, no variation was identified between the two parental-derived sequence haplotypes. Due to the residual presence of heat-resistant DNA polymerase and dNTPs which could perform end-filling and adenine-tailing reactions during heat-inactivation of MspJI, the DNA fragments from restriction enzyme digestion could be directly used for DNA adapter ligation (Figure 6d). Cost assumption analysis suggested that the MspJI digestion method would be less expensive than the previously described costreduced physical shearing method (Additional file 10) [22]. Due to a lesser requirement for capital expenditure on

Table 2 Resequencing results of the BAC clones

\begin{tabular}{|c|c|c|c|c|c|c|c|c|}
\hline \multirow[b]{2}{*}{$\begin{array}{l}\text { BAC } \\
\text { name }\end{array}$} & \multirow[b]{2}{*}{$\begin{array}{l}\text { BAC } \\
\text { length }\end{array}$} & \multicolumn{4}{|c|}{ All reads } & \multicolumn{3}{|c|}{100,000 read subset } \\
\hline & & $\begin{array}{l}\text { Total } \\
\text { reads }\end{array}$ & $\begin{array}{l}\text { Aligned } \\
\text { reads }(\%)\end{array}$ & $\begin{array}{l}\text { Ratio of } \\
\text { coverage (\%) }\end{array}$ & $\begin{array}{l}\text { Average coverage } \\
\text { depth (time) }\end{array}$ & $\begin{array}{l}\text { Aligned } \\
\text { reads }\end{array}$ & $\begin{array}{l}\text { Ratio of } \\
\text { coverage (\%) }\end{array}$ & $\begin{array}{l}\text { Average coverage } \\
\text { depth (time) }\end{array}$ \\
\hline MXK3 & $81 \mathrm{~kb}$ & 395,498 & $27,585(7.0 \%)$ & $99.6 \%$ & 82.3 & 7086 & $98.6 \%$ & 21.3 \\
\hline F20D21 & 143 kb & 558,276 & $86,861(15.6 \%)$ & $99.8 \%$ & 149.4 & 15382 & $99.2 \%$ & 26.5 \\
\hline F20B17 & $90 \mathrm{~kb}$ & 478,300 & 46,409 (9.7\%) & $>99.9 \%$ & 126.2 & 9173 & $99.9 \%$ & 24.9 \\
\hline
\end{tabular}

Ratio of coverage denotes the value for specific nucleotides located on each clone. A subset of 100,000 reads was obtained using the Seqtk software. 

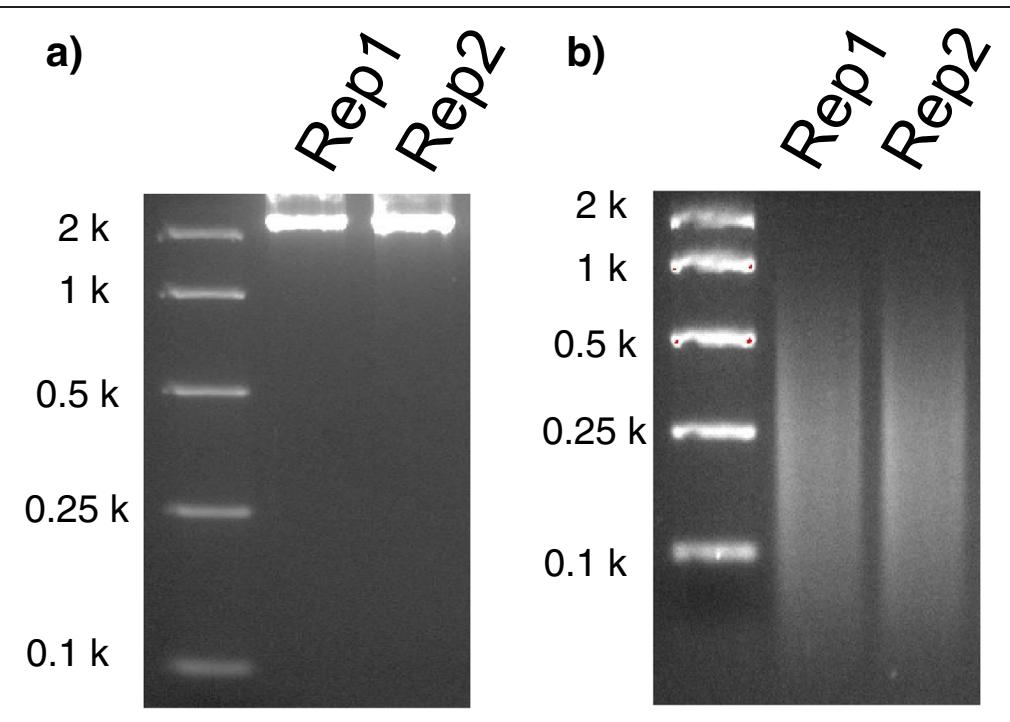

c)

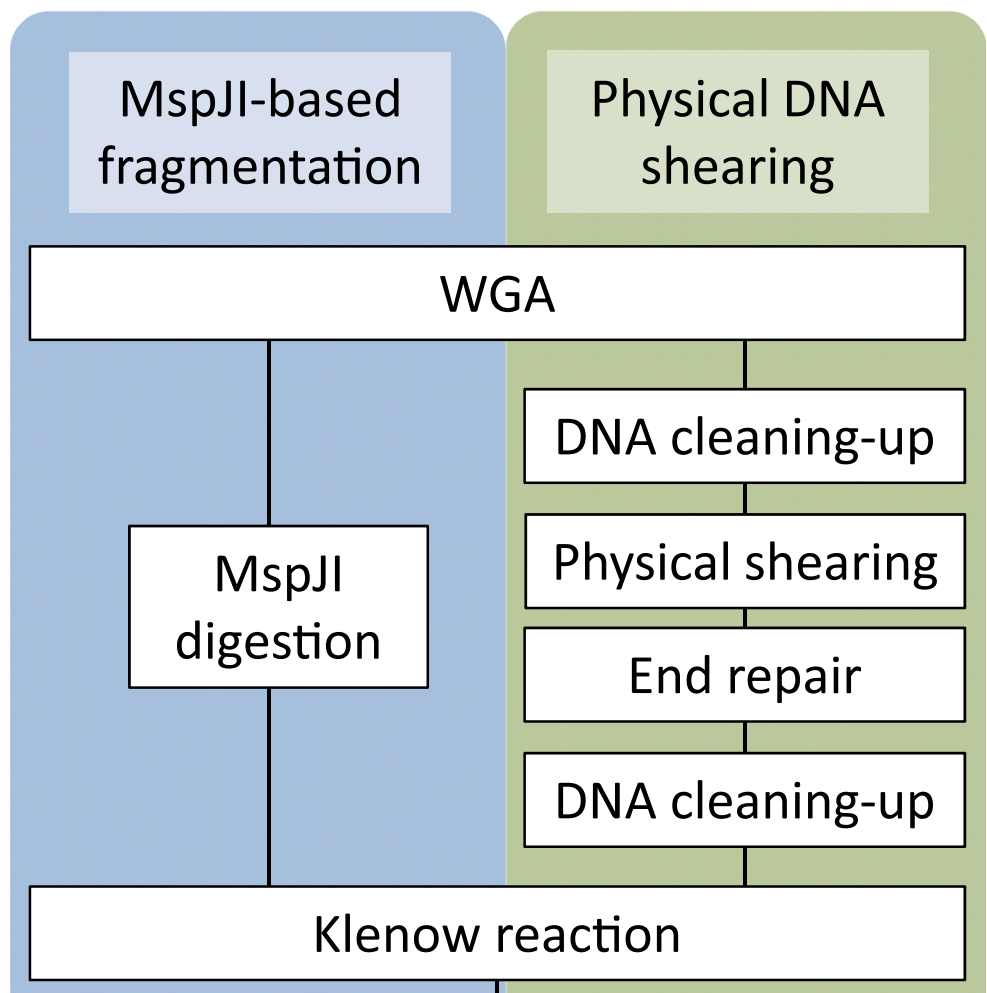

\section{Adapter ligation and following library prep procedures}

Figure 5 Sequencing of the fungal endophyte genome with the MspJl-based DNA fragmentation method. Two independent experiments (rep1 and rep2) were performed using the single endophyte strain. (a) WGA of the endophyte genome with the $\Phi 29$ enzyme in the presence of 5-methyl-dCTP $(X \mu M)$. (b) MspJl digestion of the endophyte genome-derived amplicons. (c) Flowchart of the MspJl- and physical shearing-based library prep procedures from WGA products. 
Table 3 Resequencing results of the ryegrass endophyte genome

\begin{tabular}{|c|c|c|c|c|c|c|}
\hline \multicolumn{2}{|l|}{ Reference } & \multicolumn{5}{|l|}{ Alignment } \\
\hline UI & Length (bp) & Aligned reads & $\begin{array}{l}\text { Ratio of } \\
\text { coverage (\%) }\end{array}$ & $\begin{array}{l}\text { Average coverage } \\
\text { depth (time) }\end{array}$ & $\begin{array}{l}\text { Max coverage } \\
\text { depth (time) }\end{array}$ & Mismatch (\%) \\
\hline gi|347366940 & $1,598,175$ & 81,237 & 88.7 & 10.1 & 749 & 1.5 \\
\hline gi|347366939 & $1,323,136$ & 59,445 & 86.7 & 8.8 & 304 & 1.4 \\
\hline
\end{tabular}

UI denotes the unique identifier of the NCBI GenBank. Short read sequencing data from two experiments (rep1 and rep2) were combined for read alignment.

equipment, the MspJI digestion method would become further cost-competitive when sample number is less than $100,000[22,23]$. The operator-specific time for the MspJI digestion method was expected to be $40 \%$ less than the physical shearing method (Additional file 11).

\section{Conclusions}

The present study has reported a novel method for DNA fragmentation using the MspJI enzyme, which has been exemplified for a range of template types. A DNA sample preparation procedure, such as buffer replacement and DNA concentration adjustment, is not essential for MspJI digestion, which permits a simple DNA library preparation procedure from amplicons. A modified method involving combined use with other modification-dependent restriction enzymes may improve the random nature of the fragmentation. The size range of the resulting fragments was capable of control through adjustment of the 5-methyl-dCTP concentration in the amplification reaction solution, providing various fragment ranges from $<100 \mathrm{bp}$ to $>2 \mathrm{~kb}$. The method may hence be applicable for recombinant DNA purposes other than second-generation massively parallel short read sequencing technologies. Development of a computational methodology may improve sequencing efficiency with this method, through optimisation of 5-methyl-dCTP concentration and prediction of coverage for each nucleotide.

\section{Methods}

\section{DNA sample preparation}

DNA samples were prepared using the QIAGEN DNeasy kit (QIAGEN, Hilden, Germany) (Arab gDNA, PsgDNA and LpgDNA) and the Gentra PUREGENE ${ }^{\circ}$ DNA Purification Kit (QIAGEN) (BtgDNA), BioRad AquaPure Genomic DNA Kit (Bio-Rad Laboratories, CA, USA) (Agro gDNA). The Soil DNA sample was extracted with the MoBio Powersoil kit (MoBio, CA, USA), following a modified protocol [24]. DNA concentrations were adjusted to 5$30 \mathrm{ng} / \mu \mathrm{l}$ in the TE buffer using the NanoDrop system (Thermo Fisher Scientific, MA, USA).

\section{DNA amplification with 5-methyl-dCTP}

Locus-specific primers were prepared for amplification of the Agro_gc40, Agro_gc50 and Agro_gc60, BtKIT1-10 and BtKIT27-37 sequences (Additional file 1), and PCR was performed with Phusion Hot Start DNA polymerase (Thermo Fisher Scientific) and The Expand Long Range dNTPack (Roche Applied Science, Penzberg, Germany), following the manufacturer's protocol. 5-methyl-dCTP (TriLink, CA, USA) was added to the PCR mixture at final concentrations of from 0 to $60 \mu \mathrm{M}$. WGA was performed using the REPLI-g mini kit (QIAGEN). Following the manufacturer's protocol, $2.5 \mu \mathrm{l}$ DNA (12.5-75 ng) was denatured with the D1 solution for three minutes, and then neutralised with the N1 solution. The amplification was performed in the reaction mixture with the presence of from 0 to $100 \mu \mathrm{M} 5$ methyl-dCTP (final concentrations) at $30^{\circ} \mathrm{C}$ for 16 hours. After incubation, the DNA polymerase was heatinactivated, and the products were diluted with the same amount of water.

\section{Restriction enzyme digestion}

The amplified DNA $(5 \mu \mathrm{l})$ was digested with $3 \mathrm{U}$ of the MspJI, FspEI or LpnPI restriction enzyme (NEB) following manufacturer's protocol. After incubation at $37^{\circ} \mathrm{C}$ for 4-16 hours, the enzyme was heat-inactivated at $70^{\circ} \mathrm{C}$ for 20 minutes.

\section{Illumina sequencing library construction}

Sequencing libraries were constructed with the TruSeq DNA Sample Preparation kit (Illumina) or NEBNext ${ }^{\circ}$ DNA Library Prep Master Mix Set for Illumina ${ }^{\circ}$ (NEB) with modifications. End-filling and adenine-tailing reactions for the MspJI-digested REPLI-g products were performed with the A-Tailing Mix or Klenow Fragment $\left(3^{\prime} \rightarrow 5^{\prime}\right.$ exo $\left.^{-}\right)$. For the PCR amplicons with the Expand Long Range dNTPack, the heat-inactivation procedure of the restriction enzyme also permitted end-filling of the restriction enzyme-digested fragments in the presence of the activated heat-resistant DNA polymerase and dNTPs. Blunt-ended DNA was purified with the AMPure XP bead kit (Life Technologies, CA, USA) and was used for the adenine-tailing reaction of the NEB kit. The 'Purify Ligation Products' process based on agarose gel electrophoresis (Illumina kit) was not performed. Following ligation of the DNA adapter index, the ligated DNA was purified with the AMPure XP bead kit and enriched through PCR. The size range of enriched DNA fragments was determined with the Agilent 2100 


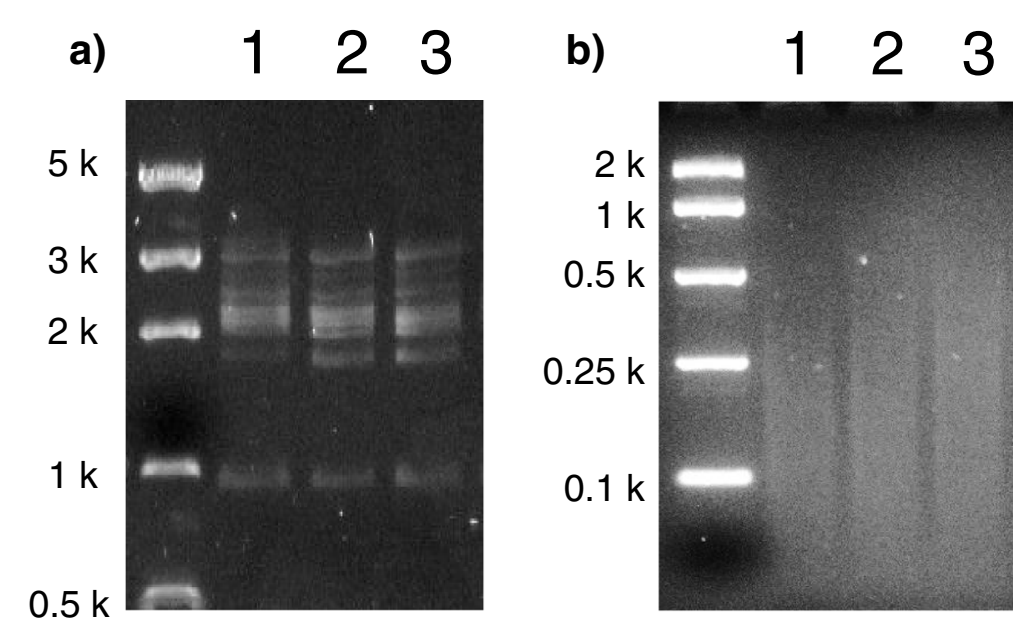

c)

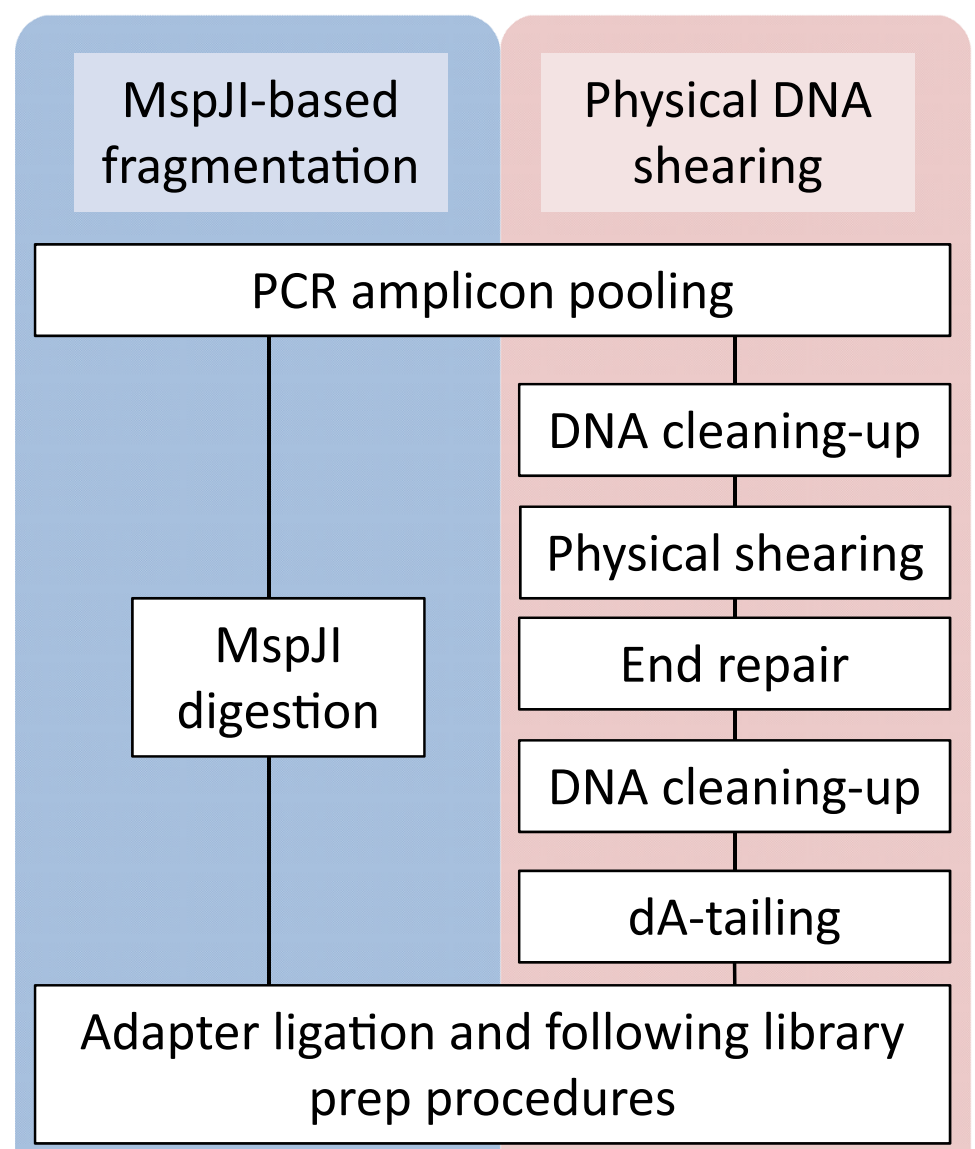

Figure 6 Resequencing of PCR amplicons with the Mspl-based DNA fragmentation method. (a) Pooled PCR amplicons containing $5^{\mathrm{m} C \text {. }}$ Locus-specific amplification was performed for each sequence independently. (b) Mspll digestion of the PCR amplicons. (c) Flowchart of the MspJI- and physical shearing-based library prep procedures from PCR amplicons. 
Bioanalyzer and Agilent DNA 1000 Kit (Agilent Technologies, CA, USA). The sequencing library was quantified with the KAPA Library Quantification Kit (Kapa Biosystems, MA, USA), following the manufacturer's protocol.

Following the standard procedure, sequencing libraries from the Agro gDNA and Arab gDNA templates were prepared with the Illumina and NEB library prep kits, respectively. For these libraries, genomic DNA was fragmented with the S2 focused-ultrasonicator system (Covaris, MA, USA) following the manufacturer's protocol.

\section{Sequencing library preparation from BAC-containing clone glycerol stocks}

Arabidopsis BAC clones (MIXK3, F20D21 and F20B17) were amplified with the QIAGEN REPLI-g mini kit. Glycerol stock $(4 \mu \mathrm{l})$ of a BAC-containing $E$. coli clone was mixed with the Buffer D1 $(4 \mu \mathrm{l})$ and incubated on ice for 5 minutes. The Buffer N1 $(8 \mu \mathrm{l})$ was added into the sample and mix by stirring with a tip. The sample was incubated at room temperature for 3 minutes. The reaction mixture, consists of $5.8 \mu \mathrm{l}$ REPLI-g Reaction Buffer, $0.2 \mu \mathrm{l} \mathrm{REPLI-g} \mathrm{Mini} \mathrm{DNA} \mathrm{Polymerase} \mathrm{and} \mathrm{0.4} \mu \mathrm{l}$ 5methyl-dCTP $(750 \mu \mathrm{M})$, was added into $3.4 \mu \mathrm{l}$ denatured sample, and the reaction mixture was incubated at $30^{\circ} \mathrm{C}$ for 16 hours. The amplicons $(5 \mu \mathrm{l})$ were digested with MspJI and the end-filling reaction was performed with Klenow Fragment $\left(3^{\prime} \rightarrow 5^{\prime}\right.$ exo $\left.{ }^{-}\right)$. Sequencing adopter ligation was performed with T4 ligase (NEB) and ligated DNA was cleaned with AMPure XP bead solution (x0.8) to exclude short DNA. DNA fragments were subsequently enriched through PCR with the phusion DNA polymerase Kit. Small fragments $(<500 \mathrm{bp})$, in which fraction E. coli genome-derived fragments were highly prevalent, were removed through size-selection with AMPure XP bead solution ( $\mathrm{x} 0.6)$. The sequencing library was characterised with the Agilent 2100 Bioanalyzer, Agilent DNA 1000 Kit, and the Qubit ${ }^{\circ}$ Fluorometer (Life Technologies), following the manufacturer's protocols.

\section{Whole genome amplification and sequencing library preparation from perennial ryegrass-derived endophyte mycelium}

Ryegrass endophyte genomic DNA was amplified with the QIAGEN REPLI-g mini kit. A section $\left(2-3 \mathrm{~mm}^{2}\right)$ of endophyte mycelium was placed into $6 \mu \mathrm{l}$ PBS solution. The Buffer D2 $(7 \mu \mathrm{l})$ was added into the sample and incubated on ice for 10 minutes, following mixing by a vortex. The Stop Solution was, then, added and mixed by a vortex. The reaction mixture, consisting of $29 \mu \mathrm{l}$ REPLI-g Reaction Buffer, $1 \mu \mathrm{l}$ REPLI-g Mini DNA Polymerase and $1 \mu \mathrm{l} 5$-methyl-dCTP $(750 \mu \mathrm{M})$, was added into $9 \mu \mathrm{l}$ of denatured sample, and the sample was incubated at $30^{\circ} \mathrm{C}$ for 16 hours. The amplicons $(5 \mu \mathrm{l})$ were digested with MspJI and the sequencing library preparation was performed following the BAC clone sequencing protocol. The PCR-enriched DNA was cleaned with AMPure XP bead solution (x0.8). The sequencing library was characterised with the Agilent 2100 Bioanalyzer and Qubit ${ }^{\circ}$ Fluorometer.

\section{Sequencing library preparation from $P C R$ amplicon}

Locus-specific primers for the $L p \mathrm{AP} 1, L p C O, L p C R Y 1$, $L p \mathrm{FLD}, L p \mathrm{FT}$, $L p L H Y$, $L p$ PHYC, $L p$ TOC1 and $L p V \operatorname{rn} 5$ sequences, and the $\mathrm{MyFi}^{\mathrm{Tu}}$ DNA Polymerase kit (BIOLINE), which contains DNA polymerase that lacks $3^{\prime} \rightarrow 5^{\prime}$ exonuclease activities, were used for PCR amplification (Additional file 1). The DNA samples of the p150/112 $F_{1}$ mapping population were used as DNA templates. In the PCR solution, $8 \mu \mathrm{M} 5$-methyl-dCTP was included [25]. The PCR products were pooled for MspJI digestion $\left(37^{\circ} \mathrm{C}\right.$ for 4 hours) and MspJI was inactivated through incubation at $70^{\circ} \mathrm{C}$ for 20 mins. The heat-inactivation procedure also permitted end-filling and adenine-tailing of the MspJI-digested fragments in the presence of the activated heat-resistant DNA polymerase and dNTPs. Sequencing adapter ligation was performed with T4 ligase and ligated DNA was cleaned with AMPure XP bead solution (x0.8) to remove short DNA. DNA fragments were, then, enriched through PCR and the product was cleaned with AMPure XP bead solution ( $\mathrm{x} 0.8)$. The sequencing library was characterised with the 2200 TapeStation system (Agilent) and Qubit ${ }^{\circ}$ Fluorometer.

\section{Massively parallel sequencing and read assembly}

The Illumina MiSeq sequencing platform was used to generate sequence output for sequencing libraries with the Illumina MiSeq Reagent Kit v2 or v3. Reads were attributed by the use of sample-specific DNA bar codes. The generated sequence reads were then checked for quality and integrity using a custom PERL script. Any reads with more than 3 consecutive Ns or more than 3 nucleotides with PHRED score $\leq 20$ or a median PHRED score $<20$ or a read length $<50$ nucleotides were trimmed or removed. The specific DNA sequence reads were then reference-aligned to the respective amplicon, Agrobacterium C58 (NCBI accession numbers: AE007869 and AE007870) [26] or Arabidopsis Columbia sequence [27] (http://www. arabidopsis.org/index.jsp). Reference alignments were performed using the BWA software package and then converted to a sorted BAM file using the SAMtools software package (http://samtools.sourceforge.net/). The Seqtk software package was used for generation of a subset sequence data (https://github.com/lh3/seqtk). Alignment of the sequencing reads to the reference sequences was visualised using the Tablet software [28]. 


\section{Availability of supporting datasets}

The data sets supporting the results of this article are included within the article and its additional files.

\section{Additional files}

Additional file 1: DNA sequence name, sequence of locus-specific PCR primers, size and G/C content ratio of PCR amplicons, and type of DNA polymerase used for PCR amplification.

Additional file 2: MspJl-enzymatic digestion of $5^{\mathrm{m}} \mathrm{C}$-containing PCR amplicons. The Agro_gc50 sequence was amplified with the Immolase ${ }^{\mathrm{TM}}$, MangoTaq ${ }^{\text {TM }}$ Pfu and Phusion Hot Start polymerases and digested with Mspll. Molar concentration denotes the 5-methyl-dCTP-concentration in the PCR solution.

Additional file 3: Characterisation of MspJ enzymatic activity.

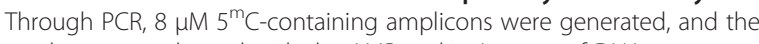
products were cleaned with the AMPure kit. A range of DNA amounts $(100,200,500$, and 1,000 ng) was used for Mspll digestion, of which $50 \mathrm{ng}$ digested DNA was visualised on an agarose gel.

Additional file 4: Illumina MiSeq sequencing libraries constructed with the MspJI, FspEl or LpnPI-digested amplicons. Sharp peaks with red and blue triangles show size standards (15 bp and 1,500 bp, respectively) of the Agilent DNA 1000 Kit. A peak between 200 and $1,500 \mathrm{bp}$ represents the size distribution of DNA fragments of the constructed DNA library.

Additional file 5: MspJI (a), FspEI (b) and LpnPI (c)-enzymatic digestion of $5^{\mathrm{m}} \mathrm{C}$-containing long PCR amplicons. Molar concentration denotes the 5-methyl-dCTP-concentration in PCR solution.

Additional file 6: Information on Arabidopsis thaliana genome-derived BAC clones used for short-read sequencing.

Additional file 7: Cost assumption (US\$) for Sanger method-based BAC-end sequencing, and $\mathbf{\Phi 2 9}$ and MspJl-based short-read sequencing. Cost for BAC-end sequencing was calculated based on previous publications [13]

Additional file 8: Illumina MiSeq sequencing library constructed with the MspJl-digested PCR amplicons. A peak between the lower and upper markers represents the size distribution of DNA fragments of the constructed DNA library.

Additional file 9: Genetic linkage mapping result of perennial ryegrass flowering signaling pathway-related sequences. The linkage maps were generated with the JoinMap ${ }^{\oplus} 3.0$ program. The LpFLD Lp Vrn5, LpTOC1, and LpLHY loci were assigned to linkage groups (LGS) 2, 5,6 , and 7 , respectively. Two loci ( $L p A P 1$ and $L p P H Y$ ) were assigned to LG4. The loci mapped through the MspJl-based fragmentation method are indicated in red.

Additional file 10: Cost assumption for Illumina sequencing library preparation. Cost (US\$) for major kit, reagent, consumable items and capital equipment was calculated based on previous publications [22,23].

Additional file 11: Time period requirement assumption for Illumina sequencing library preparation. Time periods required for library construction with the Covaris equipment-based and MspJl-based DNA fragmentation methods were estimated based on previous publications [22]

\section{Competing interests}

The authors declare that they have no competing interests.

\section{Authors' contributions}

HS performed the experimental work described in the study, co-conceptualised the study and prepared the manuscript. MS, AM, PK, and Y-HL assisted in performance of the experimental work. NOIC contributed to interpretation of data, co-conceptualised the study and contributed to preparation of the manuscript. GCS and JWF co-conceptualised the study and contributed to preparation of the manuscript. All authors read and approved the final manuscript.

\section{Acknowledgments}

This work was supported by funding from the Victorian Department of Economic Development, Jobs, Transport and Resources and the Dairy Futures Cooperative Research Centre (DFCRC). The authors would like to acknowledge Drs. Amanda Chamberlain, Tony Gendall, Helen Hayden Jatinder Kaur and Sukhjiwan Kaur, Ms. Joanne Rachel Ernest, Mr. Brett Mason and Mrs. Shimna Sudheesh for provision of the DNA samples, and plant and fungal materials. We would also like to acknowledge Mr. Sami Hakim for assistance during the sequencing process.

\section{Author details}

'Department of Economic Development, Jobs, Transport and Resources, Biosciences Research Division, AgriBio, Centre for AgriBioscience, 5 Ring Road, La Trobe University Research and Development Park, Bundoora, Victoria 3083, Australia. ${ }^{2}$ Dairy Futures Cooperative Research Centre, Bundoora, Australia. ${ }^{3}$ School of Applied Systems Biology, La Trobe University, Bundoora, Victoria 3086, Australia.

Received: 11 February 2015 Accepted: 30 March 2015

Published online: 11 April 2015

\section{References}

1. Poptsova MS, Il'icheva IA, Nechipurenko DY, Panchenko LA, Khodikov MV, Oparina NY, et al. Non-random DNA fragmentation in next-generation sequencing. Sci Rep. 2014;4:4532.

2. Knierim E, Lucke B, Schwarz JM, Schuelke M, Seelow D. Systematic comparison of three methods for fragmentation of long-range PCR products for next generation sequencing. PLoS One. 2011;6:e28240

3. Syed F, Grunenwald H, Caruccio N. Optimized library preparation method for next-generation sequencing [advertising feature]. Nat Methods. 2009;6:i-ii.

4. Syed F, Haiying G, Nicholas C. Next-generation sequencing library preparation: simultaneous fragmentation and tagging using in vitro transposition [advertising feature]. Nat Methods. 2009;6:i-ii.

5. Zheng Y, Cohen-Karni D, Xu D, Chin HG, Wilson G, Pradhan S, et al. A unique family of Mrr-like modification-dependent restriction endonucleases. Nucleic Acids Res. 2010;38:5527-34

6. Cohen-Karni D, Xu D, Apone L, Fomenkov A, Sun Z, Davis PJ, et al. The Mspll family of modification-dependent restriction endonucleases for epigenetic studies. Proc Natl Acad Sci. 2011;108:11040-5.

7. Chen W-H, Qin Z-J, Wang J, Zhao G-P. The MASTER (methylation-assisted tailorable ends rational) ligation method for seamless DNA assembly. Nucleic Acids Res. 2013;41:e93.

8. Wong KK, McClelland M. PCR with 5-methyl-dCTP replacing dCTP. Nucleic Acids Res. 1991;19:1081-5.

9. Wong KK, Markillie LM, Saffer JD. A novel method for producing partia restriction digestion of DNA fragments by PCR with 5-methyl-CTP. Nucleic Acids Res. 1997:25:4169-71.

10. Mascher M, Stein N. (2014) Genetic anchoring of whole-genome shotgun assemblies. Frontiers in Genetics 5. doi:10.3389/fgene.2014.00208

11. Lee $\mathrm{KC}, \mathrm{T}-\mathrm{H} \mathrm{CRO}$, Robertson JS, Pierce GJ, Paterson AH. A genome-wide BAC end-sequence survey of sugarcane elucidates genome composition, and identifies BACs covering much of the euchromatin. Plant Mol Biol. 2013;81:139-47. doi:10.1007/s11103-012-9987-x.

12. Breen J, Wicker T, Shatalina M, Frenkel Z, Bertin I, Philippe R, et al. A Physical Map of the Short Arm of Wheat Chromosome 1A. PLoS One. 2013;8:e80272 doi:10.1371/journal.pone.0080272.

13. Kim H, Miguel PS, Nelson W, Collura K, Wissotski M, Walling JG, et al. Comparative Physical Mapping Between Oryza sativa (AA Genome Type) and O. punctata (BB Genome Type). Genetics. 2007;176:379-90. doi:10.1534/genetics.106.068783.

14. Mira A, Martín-Cuadrado AB, D’Auria G, Rodríguez-Valera F. (2010) The bacterial pan-genome: a new paradigm in microbiology. International Microbiology 45-57. doi:10.2436/20.1501.01.110

15. Stajich JE, Harris T, Brunk BP, Brestelli J, Fischer S, Harb OS, et al. FungiDB: an integrated functional genomics database for fungi. Nucleic Acids Res. 2012:40:D675-81. doi:10.1093/nar/gkr918.

16. Lee J, Young JP. The mitochondrial genome sequence of the arbuscular mycorrhizal fungus Glomus intraradices isolate 494 and implications for the phylogenetic placement of Glomus. New Phytol. 2009;183:200-11. 
17. Larkin DM, Daetwyler HD, Hernandez AG, Wright CL, Hetrick LA, Boucek L, et al. Whole-genome resequencing of two elite sires for the detection of haplotypes under selection in dairy cattle. Proc Natl Acad Sci. 2012;109:7693-8.

18. Huang X, Wei X, Sang T, Zhao Q, Feng $Q$, Zhao Y, et al. Genome-wide association studies of 14 agronomic traits in rice landraces. Nat Genet. 2010;42:961-7.

19. Mamanova L, Coffey AJ, Scott CE, Kozarewa I, Turner EH, Kumar A, et al. Target-enrichment strategies for next-generation sequencing. Nat Methods. 2010;7:111-8.

20. Fritsche S, Wang X, Li J, Stich B, Kopisch-Obuch F, Endrigkeit J, et al. A candidate gene-based association study of tocopherol content and composition in rapeseed (Brassica napus). Front Plant Sci. 2012;3:129.

21. Jones ES, Mahoney NL, Hayward MD, Armstead IP, Jones JG, Humphreys MO, et al. An enhanced molecular marker based genetic map of perennial ryegrass (Lolium perenne) reveals comparative relationships with other Poaceae genomes. Genome. 2002;45:282-95.

22. Rohland N, Reich D. Cost-effective, high-throughput DNA sequencing libraries for multiplexed target capture. Genome Res. 2012;22:939-46.

23. Lennon NJ, Lintner RE, Anderson S, Alvarez P, Barry A, Brockman W, et al. A scalable, fully automated process for construction of sequence-ready barcoded libraries for 454. Genome Biol. 2010;11:R15.

24. Hayden HL, Mele PM, Bougoure DS, Allan CY, Norng S, Piceno YM, et al. Changes in the microbial community structure of bacteria, archaea and fungi in response to elevated $\mathrm{CO}_{2}$ and warming in an Australian native grassland soil. Environ Microbiol. 2012;14:3081-96.

25. Shinozuka H, Cogan NO, Spangenberg GC, Forster JW. Quantitative Trait Locus (QTL) meta-analysis and comparative genomics for candidate gene prediction in perennial ryegrass (Lolium perenne L.). BMC Genet. 2012;13:101.

26. Goodner B, Hinkle G, Gattung S, Miller N, Blanchard M, Qurollo B, et al. Genome sequence of the plant pathogen and biotechnology agent Agrobacterium tumefaciens C58. Sci (New York). 2001;294:2323-8.

27. Initiative AG. Analysis of the genome sequence of the flowering plant Arabidopsis thaliana. Nature. 2000;408:796-815.

28. Milne I, Stephen G, Bayer M, Cock PJA, Pritchard L, Cardle L, et al. Using Tablet for visual exploration of second-generation sequencing data. Brief Bioinform. 2012;14:193-202.

\section{Submit your next manuscript to BioMed Central and take full advantage of:}

- Convenient online submission

- Thorough peer review

- No space constraints or color figure charges

- Immediate publication on acceptance

- Inclusion in PubMed, CAS, Scopus and Google Scholar

- Research which is freely available for redistribution 